\title{
Imaging of the surface of human and bovine articular cartilage with ESEM and AFM
}

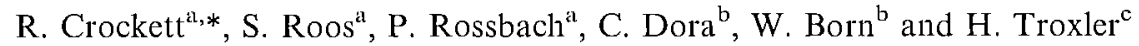 \\ "Swiss Federal Laboratories for Material Science and Technology, Ueberlandstrasse 129, 8600 Duebendorf, Switzerland \\ ${ }^{b}$ Orthopaedic University Hospital Balgrist, Forchstrasse 340, 8008 Zurich, Switzerland \\ ${ }^{c}$ University of Zurich Childrens Hospital, Steinwiesstrasse 75, 8032 Zurich, Switzerland
}

Received 16 April 2005; accepted 3 July 2005

\begin{abstract}
The surface of human and bovine articular cartilage was imaged with envirommental SEM and AFM. The effective modulus of the surface, from force-distance curves obtained with AFM, remained constant at $9 \pm 2 \mathrm{kPa}$ in the presence of synovial fluid. Extensive washing of the cartilage surface with phosphate buffered saline (PBS) removed a superficial gel-like layer, leaving a granular layer intact. Force-distance curves showed that the chemical and mechanical properties of the gel exposed to PBS changed over time. The effective modulus at the surface dropped from 481 to $4 \mathrm{kPa}$ over an hour. The results suggest that the gel-like layer, having partly lost water through evaporation on removal from the joint, absorbs water from PBS. It becomes softer and eventually begins to dissolve. The low effective modulus of the gel-like layer in synovial fluid indicates that it is too soft to influence the surface roughness. Imprints of the surface under pressure were taken using a low viscosity dental kit. Imaging of the imprint surface indicated that the topography of the cartilage under pressure was similar to that of the surface after removal of the gel-like layer. In conclusion, imaging of articular cartilage with ESEM and AFM revealed two distinct non-fibrous layers, which are granular and gel-like, and cover the fibrous collagen matrix.
\end{abstract}

KEY WORDS: cartilage, AFM, ESEM, biotribology, synovial fluid

\section{Introduction}

Synovial fluid in contact with articular cartilage provides excellent tribological properties of very low wear and friction [1]. It has been suggested that fluidfilm lubrication plays a major role in protecting articular cartilage during normal activity, whereas complementary mechanisms are required at the beginning of motion [2-4]. There have been a number of proposals for the mechanism of lubrication in articular joints under severe loading conditions or at the beginning of motion. Joint lubrication under these conditions is generally considered to be boundary lubrication, analogous to man-made systems in which the lubricating fluid is squeezed out of the contact at high pressure or low speed and the contacting surfaces are protected by surface active additives. For example, it has been proposed that phospholipids [5] or the protein, lubricin [6], are responsible for boundary lubrication in natural joints. Other theories, which deviate from classical boundary lubrication, include electrostatic repulsion [7] and the formation of a gel containing hyaluronan at the surface [8]. Kumar et al. and Sawae et al. both observed the presence of two layers, a superficial gel-like layer and a fibrous layer assigned to the collagen network of cartilage $[9,10]$. They imaged the surface of articular cartilage in solution with atomic force microscopy (AFM) before

*To whom correspondence should be addressed.

E-mail: rowena.crocketl@empa.ch and after treatment with chondroitinase $\mathrm{ABC}$, hyaluronidase and alkaline protease. Kumar et al. found that the superficial layer remained intact after treatment with chondroitinase $\mathrm{ABC}$ and hyaluronidase, and that the fibrous layer was exposed following digestion with alkaline protease [9]. Sawae et al., on the other hand, observed a fibrous network with AFM after treatment of the superficial layer with chondroitinase $A B C[10]$. Both studies proposed a gel-like surface, however, the findings on the composition of the gel were contradictory, with evidence for proteoglycans in one study and for proteins or glycoproteins in the other. With a staining technique using the cationic dye cupromeronic blue and varying concentrations of magnesium chloride, sulphated proteoglycans were undetectable in a superficial layer of $50 \mathrm{~nm}$, but were present in the adjacent non-fibrous layer of between 100 and $400 \mathrm{~nm}$ [11]. It has been proposed that a fluid film is maintained between the surfaces at low speeds by trapping water in the superficial gel layer $[12,13]$.

AFM has proved to be a powerful tool for the surface analysis of biological samples as it allows indentation measurements, as well as imaging, to be carried out in solution $[14,15]$. Indentation measurements on the macroscopic scale, using probes with diameters in the millimetre range, revealed a significant dependence of the elastic modulus of cartilage on the preparation of the sample. Values ranging from about 2 to $12 \mathrm{MPa}$ have been found for native human cartilage [16,17]. Articular 
cartilage predominantly consists of collagen type II, proteoglycans and water in a layered structure, together determining its mechanical properties [18]. Therefore, measurements of the mechanical properties on the macroscale reveal an average over a large volume. Nanoindentation with AFM records variations in the mechanical properties of the fine structure and, additionally, provides a more surface specific measurement. Stolz et al. found dynamic elastic moduli of around 21$27 \mathrm{kPa}$ for porcine articular cartilage using silicon nitride tips with a nominal radius of $20 \mathrm{~nm}$ [19]. Patel and Mao, also using sharp silicon nitride tips, found Young's moduli ranging from 0.95 to $2.34 \mathrm{MPa}$ for adult rabbit articular cartilage and a smaller range of $0.92-1.02 \mathrm{MPa}$ in neonatal rabbit articular cartilage [20].

Besides the mechanical and chemical properties of the cartilage surface, its roughness is also of tribological importance. AFM measurements of the cartilage surface revealed a mean surface roughness $(\mathrm{Ra})$ between 95 and $130 \mathrm{~nm}$ in areas of $5 \times 5 \mu \mathrm{m}$ on neonatal rabbit cartilage [20] and about $500 \mathrm{~nm}$ in areas of $100 \times 100 \mu \mathrm{m}$ on immature bovine cartilage [21].

Most AFM measurements in solution have been carried out in phosphate buffered saline (PBS), although some of the components of cartilage are soluble in this medium [22]. In the present study, AFM was used to examine effects of PBS treatment on the structure of the cartilage surface over time and to compare the mechanical properties of the superficial layer in the presence of PBS and of synovial fluid. The samples were also examined with environmental scanning electron microscopy (ESEM), before and after PBS treatment. ESEM is a technique which allows imaging of organic samples without the need for coating with gold [23]. Moreover, it does not require a high vacuum, allowing images of moist samples to be obtained. As cartilage is a viscoelastic solid [24-26], experiments were carried out to determine the effect of applied pressure on the roughness of the surface.

\section{Experimental}

Bovine articular cartilage was removed from both of the femur knee joints of five slaughtered cows younger than of 19 months of age. A punch was used to remove circular samples with a diameter of $10 \mathrm{~mm}$. The bovine samples were measured immediately after removal from the joint. Human articular cartilage was collected from femur heads that were surgically removed with written consent from four patients undergoing replacement of the hip joint at the Orthopaedic University Hospital Balgrist, Zurich. The study was approved by the Ethics Committee of the Canton Zurich. AFM measurements were carried out on macroscopically intact cartilage areas. The samples were stored at $-60{ }^{\circ} \mathrm{C}$ until analyses.

Sections through the cartilage were obtained with a sharp scalpel. ESEM (Philips ESEM-FEG XL30, FEI, Netherlands) was used to image the surface of both human and bovine articular cartilage. Human cartilage was thawed and then submersed in PBS (Fluka Chemie GmbH, Buchs, Switzerland) for 5 min before ESEM. The bovine articular cartilage was briefly washed with PBS and immediately analysed with ESEM. Alternatively, as indicated in the text, cartilage was washed in PBS at $4{ }^{\circ} \mathrm{C}$ for $5 \mathrm{~h}$ with shaking. AFM images and force-distance curves of cartilage in PBS and bovine synovial fluid were collected with Dimension 3000 SPM (Digital Instrument, Inc. Santa Barbara, California). Silicon nitride (NP-20) AFM probes (Veeco Instruments GmbH, Mannheim, Germany) with a nominal spring constant of $0.12 \mathrm{~N} / \mathrm{m}$ were used for imaging (contact mode) and force-distance measurements (figure 1). The force-distance curves, measured at a rate of $0.2 \mathrm{~Hz}$ $\left(1.2 \mu \mathrm{m} \mathrm{s}^{-1}\right)$, were fitted with the Hertz model for an infinitely stiff, cone shaped tip against soft materials: $F=\left(\pi \delta^{2} / 2\right)\left(E / 1-\eta^{2}\right) \tan (\alpha)$, where $F$ is the force, $\delta$ is the indentation depth, $E$ is Young's modulus, $\eta$ is Poisson's ratio, and $\alpha$ is the opening angle of the cone, to give the effective modulus $E^{*}=\left(E / 1-\eta^{2}\right)$ [27]. The opening angle of the cone indicated by the manufacturer was $55^{\circ}$. The spring constants were calibrated using cantilevers of known spring constant. For repulsive curves, the zero of separation was estimated as the point where the force deviated from zero. The constant compliance was calculated from the force-distance curves of each tip against a silicon wafer.

Imprints of the surface were obtained from circular samples of bovine cartilage (diameter $10 \mathrm{~mm}$ ) immediately after removal from the joint without washing. The

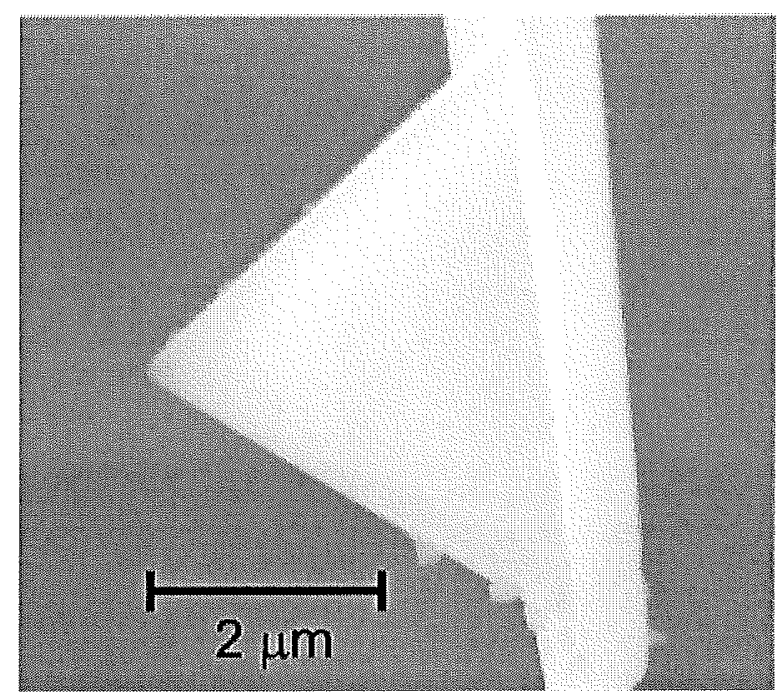

Figure 1. SEM image of an NP-20 AFM tip measured at $20 \mathrm{kV}$. 
samples were placed in a metal tube and covered with a dental kit of low viscosity (Prestige Light' ${ }^{\mathrm{TM}}$, MF Dental, Germany) and a pressure of $0.50 \mathrm{MPa}$ was applied for $10 \mathrm{~min}$, in which time the dental kit hardened. The positive imprint of the hardened dental kit was obtained with an epoxy resin (Durcupan ${ }^{\mathrm{TM}}$, Fluka Chemie GmbH, Buchs, Switzerland).

\section{Results}

\subsection{ESEM and AFM images}

ESEM of the human articular cartilage showed a smooth gel-like surface with some protrusions (figure 2(a)). When the cartilage was washed in PBS for $5 \mathrm{~h}$, the gel-like layer disappeared (figure 2(b)). The surface appeared rougher and uniform. A section through the cartilage, parallel to the surface, showed a fibrous structure with lacunae from chondrocytes (figure 2(c)).

As the cartilage samples were obtained from patients with diseased joints and may have been altered by freezing, ESEM was also carried out with bovine articular cartilage immediately after removal from the joint. As with human cartilage, a superficial gel-like layer was also observed and washed away with PBS (not shown). When the samples were left in the ESEM for more than 20 min at 0.8 Torr, the superficial layer dried out. The deformation of the surface caused by loss of water is shown in figure 2(d). The superficial gel-like layer was recovered when the samples were rehydrated in PBS for 5 min.

AFM of human articular cartilage was carried out in PBS. After thawing, the samples were preincubated in
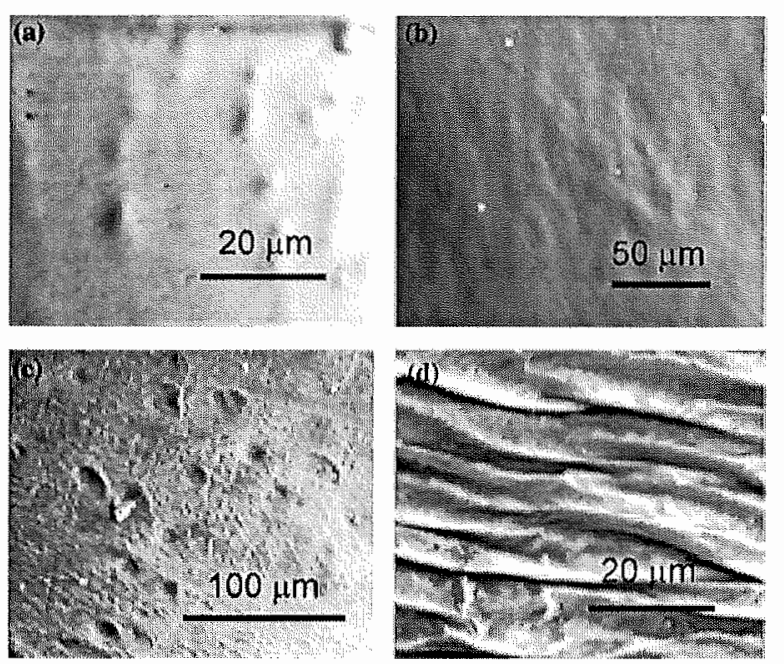

Figure 2. Environmental SEM measurements at $3 \mathrm{kV}$ and 0.8 Torr of the surface of human femur cartilage before (a) and after (b) washing with PBS. (c) Image of a section through the cartilage parallel to surface. (d) Surface of the sample shown in (a) after drying in the ESEM chamber.
PBS at room temperature for $30 \mathrm{~min}$. Strong adhesion between the tip and the cartilage surface prevented imaging of the samples immediately after thawing. AFM height and deflection images of the gel-like layer in PBS showed a uniform surface that was streaked easily by the AFM probe (figure 3(a,b)). Extensive washing of the cartilage with PBS followed by AFM revealed a more solid and granular appearance of the surface (figure 3(c, d)). Profiles taken from the centre of the height images of the cartilage before (figure 3(e)) and after (figure 3(f)) washing with PBS showed distinct topographies of the two samples [28].

Images of the bovine cartilage, measured in PBS before and after washing with PBS, were similar to those of human samples in PBS. AFM images of bovine articular cartilage, obtained in the presence of synovial fluid immediately after removal from the joint (figure 4(a)), were also similar to those of human (figure 3(a)) and bovine articular cartilage in PBS. Much like the human cartilage, a granular layer appeared on the cartilage surface upon removal of the gel-like layer with PBS (figure 4(b)). As it was not possible to obtain a microscopic image of the cartilage surface in synovial fluid, a section through the middle of the cartilage, parallel to the
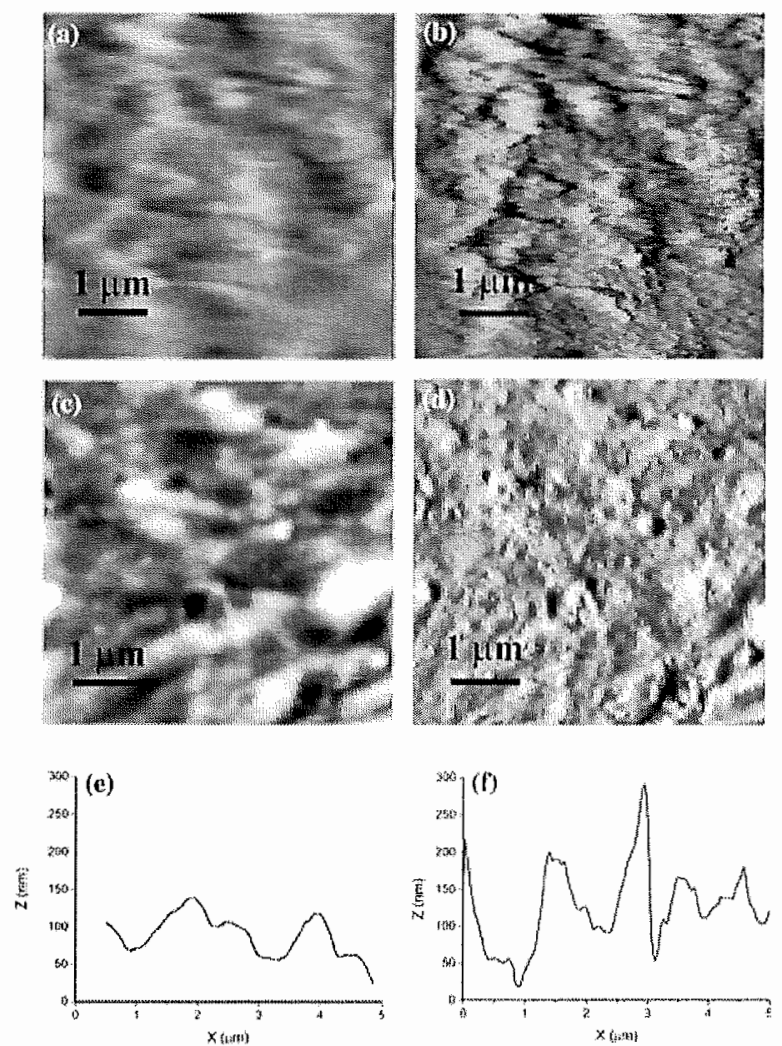

Figure 3. AFM height (a, c) and deflection (b, d) images of $5 \times 5 \mu \mathrm{m}$ on human femur cartilage in PBS, before $(a, b)$ and after $(c, d)$ washing with PBS. (e) Profile in the middle of the height image shown in (a). (f) Profile in the middle of the height image shown in (c). 

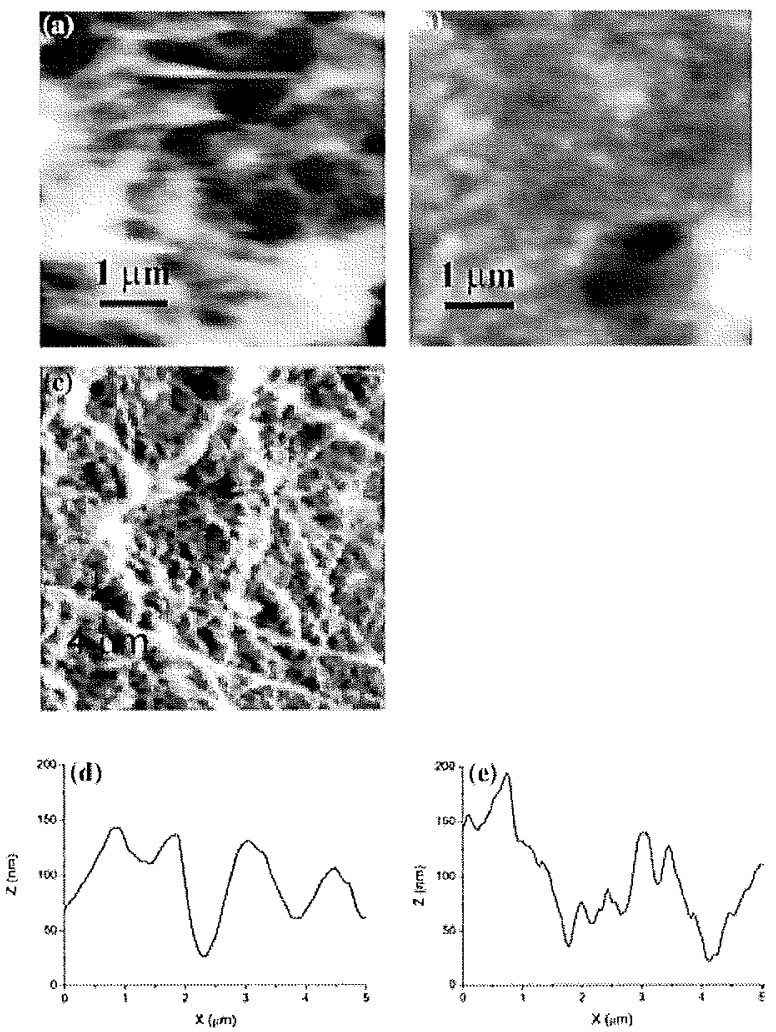

Figure 4. AFM height images of a $5 \times 5 \mu \mathrm{m}$ area of bovine cartilage in synovial fluid: surface before (a) and after (b) washing with PBS (c) Surface of a section through cartilage, parallel to surface (area $30 \times 30 \mu \mathrm{m}$, height range $700 \mathrm{~nm}$ ) (d) Profile in the middle of the height image shown in (a). (e) Profile at approximately the middle of the height image shown in (b).

surface, was imaged to ensure that the two sides of the cartilage sample could be distinguished. This image showed the structure of collagen fibres (figure 4(c)). Profiles were taken from the height images of the samples before and after a PBS wash (figure $4(d, e)$ ).

\subsection{AFM force-distance curves}

Force-distance curves were obtained for the surface of bovine articular cartilage in the presence of PBS and synovial fluid. The bovine cartilage was removed from the joint and then fixed to a silicon wafer before being submersed in PBS. During the first 20 min after submersion, strong adhesion prevented the cantilever from moving away from the surface and, as a consequence, force-distance could not be measured. The decrease in adhesion with time, reflecting changes on the cartilage surface, made sequential measurements in different areas of the same sample impossible. Therefore, seven different specimens were measured. Figure 4 shows the change in the curves for unloading over time at the beginning of the measurement. While adhesion was still strong (figure 5), a fit for the Hertz equation could not

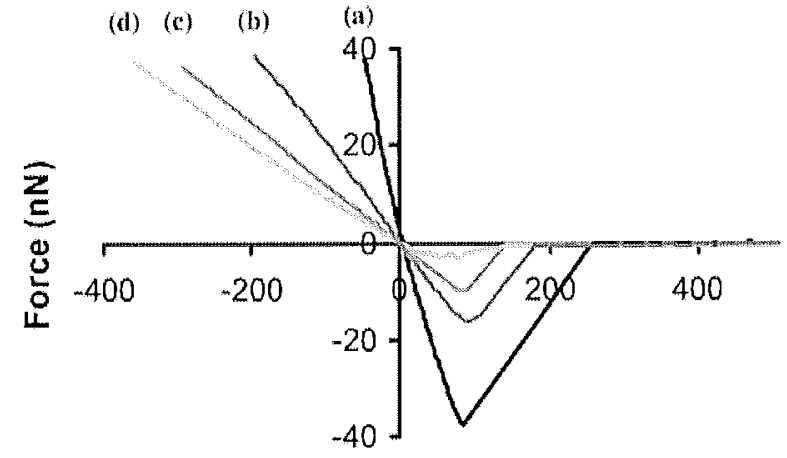

Separation $(\mathrm{nm})$

Figure 5. Unloading force-distance curves of four measurements at (a) $20 \mathrm{~min}$, (b) $22 \mathrm{~min}$, (c) 24 min, and (d) 26 min after submersion of the bovine cartilage in PBS. The indentation into the surfaces increases with decreasing adhesion.

be found as this equation does not allow for adhesion [27]. Johnson-Kendall-Roberts theory allows adhesive curves to be fitted, however the tips used in this study were closer to a conical than to a spherical shape. With time, better fits of the curves were obtained although some adhesion was still evident during unloading.

The effective modulus $\left(E^{*}=\left(E / 1-\eta^{2}\right)\right)$ of the superficial gel-like layer in PBS and the adhesion of the AFM tip to the surface decreased with time (figure 6). A small swelling occurred on the surface. As a consequence, the tip had to be removed from the surface between measurements. Finally, the force-distance curves, for loading and unloading, became repulsive and the effective modulus dropped to a very low level. The fit for the Hertz equation at this stage was very poor. For the seven samples measured, the effective modulus dropped from a maximum of $481 \mathrm{kPa}\left(R^{2}=0.996\right)$ at the beginning of the experiment to a minimum of $4 \mathrm{kPa}$ $\left(R^{2}=0.974\right)$ after $1 \mathrm{~h}$. Clearly, a number of assumptions have to be made for the Hertz equation. These include

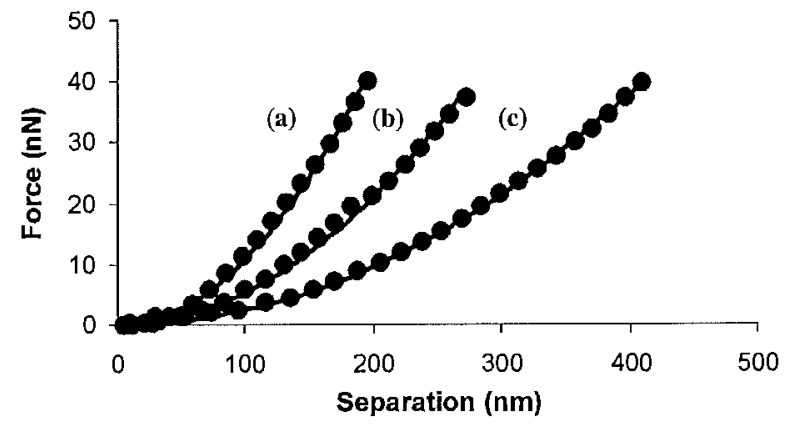

Figure 6. Force distance curves fitted with the Hertz equation for three measurements in PBS. The number of data points has been reduced for clarity. (a) $E^{*}=481 \mathrm{kPa}, R^{2}=0.996$, b) $E^{*}=234 \mathrm{kPa}$, $R^{2}=0.996$, (c) $E^{*}=106 \mathrm{kPa}, R^{2}=0.999$. 


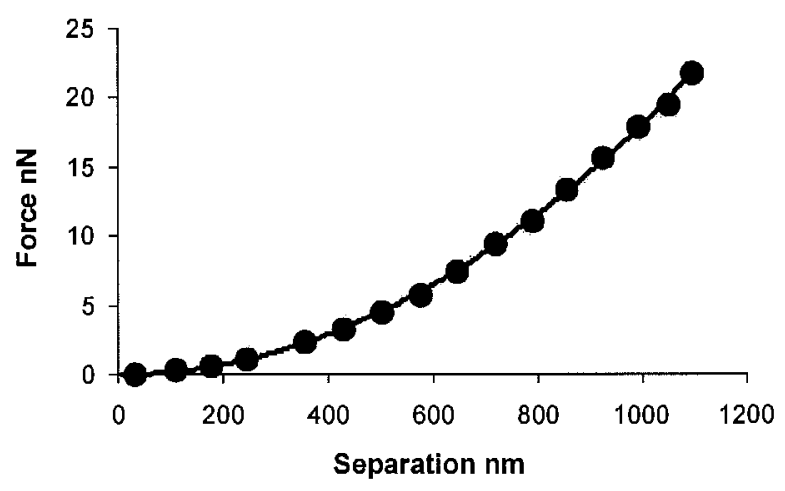

Figure 7. Fitted curve for force-distance measurement on bovine cartilage in synovial fluid. The number of data points has been reduced for clarity.

the assumption that the change in the gel-like layer during the measurement is not significant. If the drop in the effective modulus between the measurement of curves (a) and (b) in figure 6 was linear, the change in the modulus during the measurement of curve (a) would be less than $1 \mathrm{kPa}$.

Force-distance curves were also obtained for 3 positions on 5 samples of bovine articular cartilage in synovial fluid. The samples remained covered with fluid during preparation, and only the bone side was dried with a paper towel before gluing it to a silicon wafer. The curves, for loading and unloading, were repulsive, as with those recorded in PBS at longer times, and they remained constant over a period of $30 \mathrm{~min}$. The effective modulus, $E^{*}$, was $9 \pm 2 \mathrm{kPa}$ (figure 7). The thickness of the gel-like layer was estimated by forcing the tip into the layer in stages and then imaging. In this way, a thickness of $20 \pm 10 \mu \mathrm{m}$ was obtained from measurements on five samples. When the cartilage was washed with PBS, the synovial fluid was also removed. These samples were, therefore, re-submersed in the synovial fluid before force-distance measurements. The results also indicated a repulsive interaction between the AFM tip and the cartilage surface and no change with time. The effective modulus for 3 positions on 5 samples was $24 \pm 3 \mathrm{kPa}$. Attempts to increase the speed of loading resulted in curves which could not be interpreted, probably due to the high viscosity of the synovial fluid.

\subsection{AFM of imprints}

In order to determine the surface roughness of the cartilage under pressure, imprints were made with a dental kit and $0.5 \mathrm{MPa}$ applied pressure. A positive inage was obtained from the imprint with an epoxy resin. AFM images of the surface of the epoxy resin revealed a mean surface roughness of $196 \pm 39 \mathrm{~nm}$ for an area of $20 \times 20 \mu \mathrm{m}$ and $19 \pm 3 \mathrm{~nm}$ for an area of $3 \times 3 \mu \mathrm{m}$ (figure 8).

\section{Discussion}

The ESEM images of human and bovine cartilage showed three distinct layers. The ease of removal of the
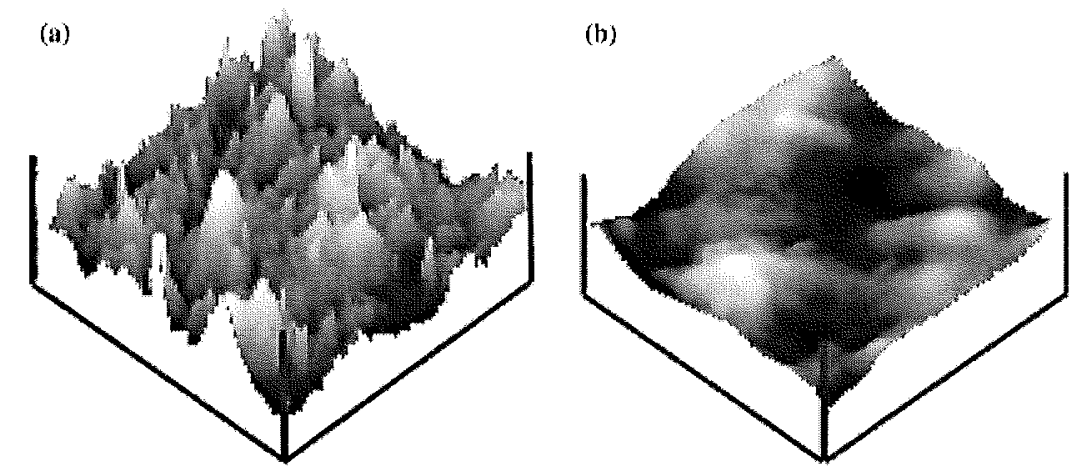

(c)

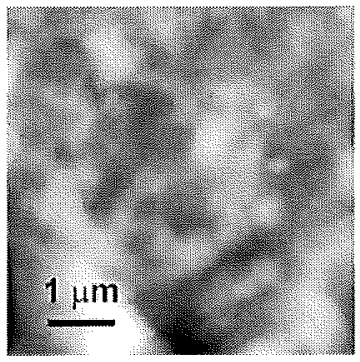

(d)

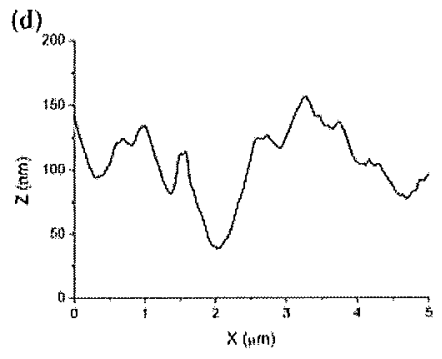

Figure 8. Representative AFM image or the epoxy resin surface derived from a dental kit imprint of the cartilage surface subjected to $0.5 \mathrm{MPa}$ pressure: (a) 3-D height image of an area of $20 \times 20 \mu \mathrm{m}$, height range $500 \mathrm{~nm}$, (b) 3-D height image of an area of $3 \times 3 \mu \mathrm{m}$, height range $100 \mathrm{~nm}$, (c) height image, area $5 \times 5 \mu \mathrm{m}$, (d) profile in the middle of the height image shown in (c). 
uppermost layer with PBS suggests that it is not part of the cartilage, but rather a separate layer, perhaps deposited from the synovial fluid or produced by cells in the cartilage. Results from a number of studies indicate a non-fibrous layer that covers the fibrous articular cartilage surface $[9,10,14]$. AFM measurements carried out by Sawae et al. were consistent with a gel-like layer with protrusions similar to those observed with ESEM. However, they found areas of granular structures on in the same surface as the gel-like layer and not on removal of the latter [10].

In the present study, imaging of articular cartilage surface with ESEM and AFM revealed three distinct layers. The uppermost layer had a gel-like appearance and was removed when the samples were thoroughly washed with PBS. The next layer showed a granular structure that covered the fibrous collagen matrix of the cartilage. AFM images of cartilage in synovial fluid also indicated a gel-like layer. This suggests that the gel-like surface of the cartilage also exists in the intact joint and was not deposited during removal from the joint or preparation in PBS. Scanning the cartilage surface in PBS with AFM required an equilibration period of 30 min to reduce an initially strong adhesion between the AFM tip and the cartilage surface. On the other hand, adhesion between the AFM tip and the surface was not observed when the cartilage specimen was submersed in synovial fluid. This indicated that the surface was sensitive to changes in the environment that occurred on removal from the joint and submersion in PBS. This was confirmed by force distance measurements over time. In the ESEM images, the gellike layer had a solid appearance and the initial AFM force-distance curves led to correspondingly high effective moduli. However, the gel-like layer became significantly softer with time in PBS, and appeared to dissolve. The effective modulus measured in synovial fluid did not change with time. It was higher than the final value found in PBS, but significantly lower than that measured immediately after the preincubation period in PBS. The low effective modulus of the cartilage in bovine synovial fluid suggests that the gel-like layer contains a large amount of water. Therefore, it is conceivable that this layer looses water after removal of synovial fluid. Subsequent rehydration with PBS would then account for the dramatic change in the effective modulus with time. The change from an adhesive to a repulsive interaction between the AFM tip and the sample also indicated a change in the chemical composition of the surface. This is consistent with the high hydrophobicity of the gel-like surface upon removal of synovial fluid [5]. The adhesion observed during measurements on cartilage in PBS can be assigned to hydrophobic adhesion, which is known to reach large values (figure 5) [29]. Hydrophobic adhesion was not observed when the cartilage was submersed in synovial fluid. This can be explained by the denaturation of proteins on the gel-like surface upon removal of the synovial fluid and their subsequent release into PBS after rehydration. Alternatively, with the loss of water, highly hydrophilic molecules in the gel-like layer may migrate towards the cartilage matrix, leaving the more hydrophobic molecules on the surface. Upon readsorption of water from PBS, this process would then be reversed. The effective modulus for the surface after removal of the gel $(24 \pm 3 \mathrm{kPa})$ in synovial fluid was similar to that found in AFM measurements on cartilage in PBS [19].

A number of techniques have been used to determine the roughness of the superficial layer of cartilage without loading [20,21]. However, as cartilage is a viscoelastic material, the structural response of the surface to pressure is of interest. Kobayashi and Oka used confocal laser scanning microscopy to analyse the surface of cartilage during compression with a glass plate [30]. They found that the surface, in areas of direct contact, appeared smooth and depressions disappeared. They also observed areas in which a fluid film separated the cartilage from the glass. In the present study, the roughness of the surface was determined on an epoxy resin image of a cartilage imprint obtained under $0.5 \mathrm{MPa}$ pressure with a low viscosity dental kit. The AFM image of the epoxy resin was similar to the granular image of the PBSwashed cartilage with the gel removed. Based on micro-elastohydrodynamic lubrication concepts, Dowson and Jin have proposed a model in which the surface roughness causes pressure perturbations [2]. These perturbations lead to a flattening of the initial asperities and result in a roughness that is significantly smaller than the predicted film thickness. A certain roughness is also a prerequisite for the microscopic flow model. Ikeuchi et al. found that fluid is initially trapped between asperities after loading [3]. They proposed that creep deformation then occurs at the contacting asperities due to flow caused by the pressure gradient from the cartilage to the space between the contacts.

Flattening of the asperities could not be determined from the image of the surface under one pressure. However, further work is being carried out to determine the effect of varying pressure on the surface roughness.

In this work, removal of the superficial gel-like layer exposed a non-fibrous surface, consistent with the observation of Orford and Gardner that there are two non-fibrous layers on top of the fibrous collagen network of articular cartilage [11]. It has been proposed that the gel-like layer traps water and prevents it from being squeezed out during loading $[12,13]$. Therefore, a fluid film is maintained between the surfaces even at low speeds. 


\section{Conclusion}

ESEM and AFM showed a soft gel-like layer on the surface of human and bovine cartilage. This layer was observed on cartilage submersed in PBS and in synovial fluid. However, the elastic modulus of the gel-like layer in PBS changed over time, and was different from that measured in synovial fluid. Therefore, it is concluded that sample storage and handling can play a critical role in the results of surface measurements. The low elastic modulus of the gel-like layer suggests that water is a predominant component. This is consistent with the theory that the mechanism of lubrication in natural joints involves the maintenance of a fluid film on the surface, analogous to boundary lubrication with polyelectrolyte brushes [12]. The observed roughness of the surface under pressure supports the models of elastohydrodynamic lubrication proposed by Dowson and Jin, and Ikeuchi [2,3]. The chemical composition of the gel-like layer remains to be analysed and further experiments are required to determine to what extent the water is forced out of the gel during loading.

\section{References}

[1] J.H. Dumbleton, Tribology of Natural and Artificial Joints (Elsevier, New York, 1981).

[2] D. Dowson and Z.-M. Jin, Eng. Med. (UK) 15(2) (1986) 63.

[3] K. Ikeuchi, M. Oka and H. Mori, Trans. Jpn. Soc. Mech. Eng. 55 (1989) 508.

[4] T. Murakami, JSME International Journal Series III-Vibration Control Engineering Engineering for Industry 33 (1990) 465.

[5] B.A. Hills, Proceedings of the Institution of Mechanical Engineers Part H-Journal of Engineering in Medicine 214 (2000) 83.

[6] D.A. Swann, F.H. Silver, H.S. Slayter, W. Stafford and E. Shore, Biochem. J. 225 (1985) 195.
[7] A.D. Roberts, Nature 231 (1971) 434

[8] A. Maroudas, Proc. Instn. Mech. Engrs. 181 (1966) 45.

[9] P. Kumar, M. Oka, J. Toguchida, M. Kobayashi, E. Uchida T. Nakamura and K. Tanaka, J. Anat. 199 (2001) 241.

[10] Y. Sawae, T. Murakami, K. Matsumoto and M. Horimolo, J. Jpn. Soc. Tribol. 45 (2000) 150.

[11] C.R. Orford and D.L. Gardner, Histochem. J. 17 (1985) 223.

[12] N. Kampr, J.F. Gohy, R. Jerome and J. Klein, J. Polym. Sci. B-Polymer Physics 43 (2005) 193.

[13] G.D. Jay, Connect. Tissue Res. 28 (1992) 71.

[14] J.S. Jurvelin, D.J. Muller, M. Wong, D. Studer, A. Engel and E.B. Hunziker, J. Struct. Biol. 117 (1996) 45

[15] M.W. Rutland, J.W.G. Tyrrell and P. Attard, J. Adhes. Sci. Technol. 18 (2004) 1199.

[16] R. Y. Hori and L.F. Mockros, J. Biomech. 9 (1976) 259.

[17] W.C. Hayes and L.F. Mockros, J. Appl. Physiol. 31 (1971) 562.

[18] C.W. Archer, Biology of Synovial Joints (Harwood Academic Publishers, Amsterdam, 1999).

[19] M. Stolz, R. Raiteri, A.U. Daniels, M.R. Van Landingham, W. Baschong and U. Aebi, Biophys. J. 86 (2004) 3269

[20] R.V. Patel and J.J. Mao, Front. Biosc. 8 (2003) A18.

[21] S. Park, K.D. Costa and G.A. Ateshian, J. Biomech. 37 (2004) 1679'.

[22] S.S. Skandalis, A.D. Theocharis, D.H. Vynios, D.A. Theocharis and N. Papageorgakopoulou, Biochimie 86 (2004) 221.

[23] K.J. McKinlay, C.A. Scotchford, D.M. Grant, J.M. Oliver, J.R. King, P.D. Brown, in: Electron Microscopy and Analysis 2003, 2004, p. 87.

[24] N.P. Cohen, R.J. Foster and V.C. Mow, J. Orthop. Sports Phys. Ther. 28 (1998) 203.

[25] W.M. Lai, J.S. Hou and V.C. Mow, J. Biomech. Eng.-Trans. ASME 113 (1991) 245.

[26] V.C. Mow, S.C. Kuei, W.M. Lai and C.G. Armstrong, J. Biomech. Eng.-Trans. ASME 102 (1980) 73

[27] G.V. Dedkov, Physica Status Solidi a-Appl. Res. 179 (2000) 3.

[28] AFM profiles were obtained with WSxM 4.0 Develop 7.5 downloaded from www.nanotec.es/download.htm.

[29] J.W. Kurutz and S.H. Xu, Langmuir 17 (2001) 7323.

[30] M. Kobayashi and M. Oka, Biomed. Mater. Eng. 13 (2003) 429. 